becomes dull and forgets all she ever thought she knew about entertaining and diverting a patient's mind, not having been able to gain a fresh view away from one personality during fifteen weeks?

Fortunately, we do not meet all these difficulties on one case very often, or many would be tempted to give up their chosen work. People wonder, even doctors, why nurses lose interest in the patient during convalescence and do not care for long cases. A nurse having a wellestablished practice wrote me that she was worn to a shred adapting herself to people. Is not that the secret of success, adapting one's self to patient and family? Here is where the personality of the woman counts for more than the skill as a nurse, though both are needed. We hear more unfavorable comments on the personality of the woman than lack of skill as a nurse. Many nurses of a few years' experience might add to the list of trials to. which I have called attention, hoping that preparation may be given coming nurses to meet these conditions.

\title{
A MODERN ITALIAN HOSPITAL
}

BI ELLEN N. LA MOTTE

Graduate of the Johns Hopkins Hospital

About fifteen years ago the Duchess of Galliera, an Italian lady of high rank, great wealth, and still greater philanthropy, founded and presented to the city of Genoa a hospital called the Hospital of St. Andrew, though its official name has been dropped by the grateful Genoese, who speak of it simply as the Galliera Hospital, in recognition of its founder. 'This institution, in point of construction, equipment, and architectural beauty, deserves to rank as one of the finest of its kind to be found in any country, and the people of Italy are justly proud of it.

It is built in the newer part of the town, and the situation leaves nothing to be desired, standing as it does on a high hill from which a splendid view of sea and harbor is obtained, and with nothing to interfere with the fresh wind as it blows in from the Mediterranean. It stands in its own grounds, and it is constructed on the pavilion plan, so that the ground space occupied by it is considerable, and gives one the impression of great size and the apparent capacity of at least three times the number of patients that it can in reality accommodate, which is rather small, having only about three hundred. The first sight of it is imposing; it stands back from the street, and the façade, built in a slight curve, is about three blocks in length and composed entirely 
of white marble. From end to end of this main front run two elaboratc marble galleries, one above the other, and the various wards, above and below, open on to them. The ward buildings or pavilions were not visible from the street, as they were at the back of this main building, to which they each joincd at right angles. There were eight of these pavilions, each two stories high and containing two wards, an uppcr and a lower, the latter being in all cases rescrved for the male paticnts. There were then sixteen wards, but only thirteen of them were in use, the other thrce, completely equipped, were kept ready for use in case of any great public calamity, such as war or pestilence.

The construction of each ward was alike in every particular, and was simple and enormously convenient, and as handsome as the lavish use of white marble could make it. On cntering from the gallery, which was the only entrance in each case, one came at once into the patients' dining-room, a large, cheerful room with a big marble table built solidly into the middle of the floor, and surrounded by stationary iron benches. As we entered the ward the patients' supper was prepared and ready for them. At each place were two hard-boiled eggs and olive oil, half a pint of red wine, a stewed pear, and a roll. The sight of the first two things gave us the start of surprise that usually follows any sudden broadening of one's horizon. The diet kitchen was to the right of the dining-room, in all respects so like those of most hospitals as to be of little interest, and contained the usual gas-stove apparatus, dumbwaiter, etc., that usually forms part of their equipment. Beyond the kitchen and between it and the ward propcr was the office of the sister in charge, a little room comfortably fitted up, and containing, besides a writing-tablc, a large medicine-closet, the use of which we did not quite sce, as in all our progress through the hospital we never oncc, by any chance, saw a dose of any sort being given to a patient. This may have been a coincidence-probably it was,-nevertheless, it was an observation we had made in every other Italian hospital we had visited, and the fact struck us again here in the Galliera. Taking temperature too was to us an unseen performance-fancy the hours of eight, twelve, and four allowed to pass without the stress of "medicincs and tempcratures"! The thing that struck us most, however, in this little officc was a small but neatly curtained window that looked directly into the main ward, and from which a splendid but guarded view of all that happened there might be obtained. One could imagine the glances that might be fired through this little port-hole-also having to work with such a veiled, omniscient eye fixed constantly upon one!

The main ward, containing about twenty-four beds, was largc, bright, and airy, well heated, and ventilated by means of little, gratcd 


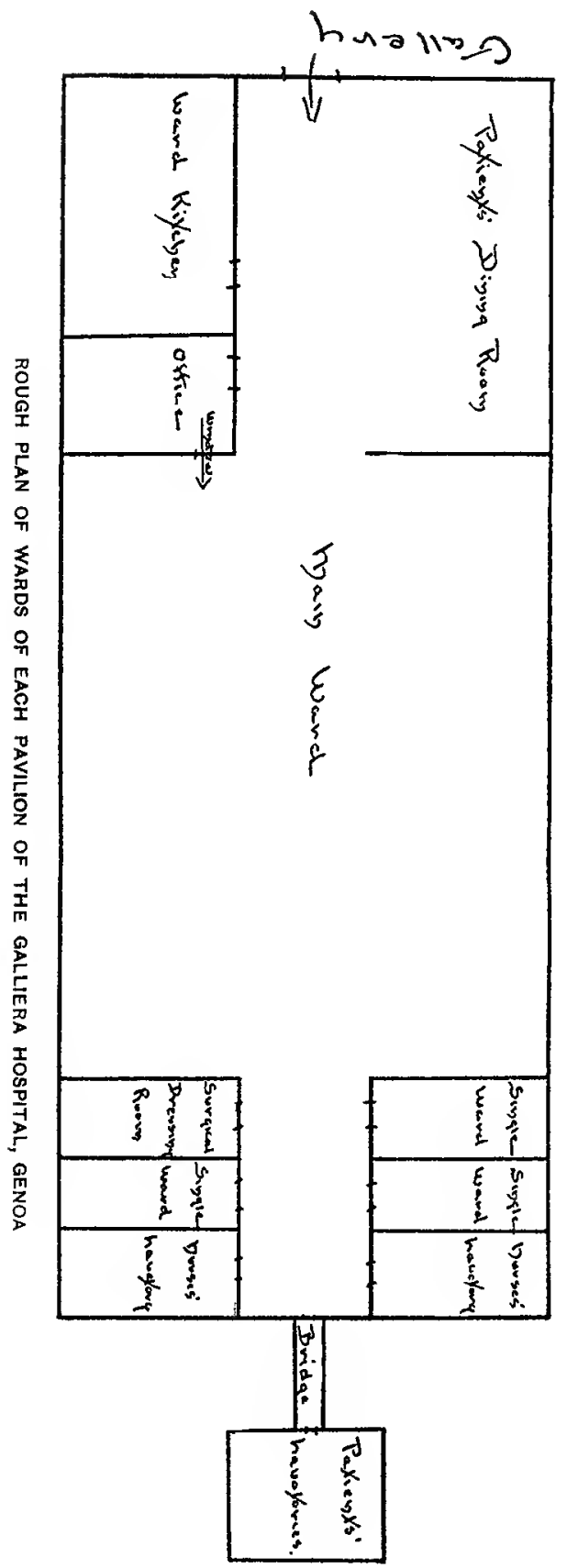


openings in the wall a few inches above floor level, and by other openings, somewhat larger, just below the ceilings. There were no screens nor wheel-chairs visible, and in the male wards all the patients wore round white cotton nightcaps with a tassel on top, the effect being rather nice. As we passed along, all the up patients stood at attention at the foot of their beds, and this bit of courtesy was required of them each time a doctor or a visitor entered the ward. Quiet and order were everywhere apparent-our entrance, however unexpected, never seemed to interrupt a song or to be a burden in any way. The sisters certainly manage well!

The main ward terminated by a narrow passage, on each side of which were three rooms; the two on the opposite sides and most distant from the ward were the lavatories for the attendants. The other rooms were small, and held one or two beds apiece for the use of either very ill patients or for those able to pay for a little extra attention. On the surgical wards, however, one of these little rooms was always used for a dressing-room in which all the daily dressings were done, even the bed patients being brought hither unless they were positively too ill to be moved. Each dressing-room had its own sterilizer, surgical carriage, cupboard for basins, bandages, etc., and on the gynæcological wards, besides the examining-table, there was an overhead row of large, brass-bound jars with rubber tubes attached, containing solutions for all the various kinds of douches, irrigations, etc., that might be needed. The great convenience and comfort, as well as the great economy of time and labor, in having such a room attached to each surgical ward is a fact that is too obvious to even require comment. The bathrooms and lavatories for the patients were not included in the ward building itself, but were placed in a separate little building, rising like a brick tower about ten feet back of each pavilion, and connccted with the wards therein by means of small, glass-cnclosed bridges. Access to them by means of these bridges was extremely simple, and their isolation completely prevented the chance of any odors from them being noticed in the wards.

The whole hospital, in every detail, seemed to be equipped with every labor-saving device that could be conceived of, and everything about it was arranged with system and mechanical detail that seemed to be most admirable. Beneath the lower of the two galleries that ran from end to end of the front of the building there were two corridors that likewise ran the full length of it. One of these, the lower corridor, was completely underground and contained all the heating, water, and lighting apparatus that was necessary to supply the various wards; thc other, or upper, corridor was above it, and lighted by windows partially below the ground level. This corridor connected at intervals 
along its length with the kitchen, pharmacy, and supply-rooms of various kinds, and the elevator of each ward opened on it. These ward elevators were also large enough for carrying patients, and when not in such use they made delivery of the daily ward supplies, such as drugs, food, linen, etc., a matter of delightfully easy accomplishment. The cars for carrying linen to and from the laundry ran on little iron tracks along this corridor, which seemed to be the main artery of the hospital, and we then followed up the tracks to the laundry itself, which is a separate building in another part of the grounds. All the machinery in it is modern; the large sterilizer for mattresses, etc., was here, and we were shown a most simple but excellent device used on all the wards for the purpose of keeping the infected linen of isolated patients separate and distinct from the general wash. This consisted simply of a large bag made of heavy brown canvas with a tightly buttoned cover, the whole thing, both in size and shape, looking for all the world like one of our United States Mail pouches. During the day the soiled linen was put in it, and every morning the bag was taken down to the laundry and its contents were there washed separately.

A mattress-making department was another feature of the institution, as, indeed, it seems to be of most of the hospitals over here, which is possibly a necessity, owing to the fact that the mattresses, when not made of wool, are made of either straw or seaweed and therefore lose their shape and become uncomfortable very rapidly. At the Galliera hospital all the mattresses were of wool, and covered with heavy white muslin instead of our more familiar striped ticking; these covers were washed and they were remade one after another from the different wards as fast as it could be accomplished. In the case of those that had been sterilized, or for any reason required immediate doing up, the usual routine was set aside and such mattresses were given precedence.

The nursing done in this hospital is on a higher plane than that of, possibly, any other Italian hospital in charge of nuns, as this is. The positions these sisters occupy is much like that of a head nurse over her particular ward or department; they look after the ward supplies and requirements, and see that the work for the patients is done, but they take no part in it themselves-their duties are practically those of an overseer. The actual nursing care of the patients, if it can be called nursing care, since our meaning of the words has acquired such a broad significance, is done by men and women of the lower classes, who are called Infirmarists. On the male wards the care is given by men Infirmarists, on the women's wards, by women, and their pay ranges from twenty-five to thirty lira (five dollars to six dollars) a month. But just here lies the difference that is to be found between these nursing servants and those of most other Italian hospitals-here 
in the Galliera therc is some slight premium placed upon intelligence, and some slight effort is being made to teach them the rudiments of practical nursing. It is true, there is no promotion beyond that which comes when the highest point of wage-earning is reached, so that the saying, "Fortune is the measure of intelligence," might here find practical application, but all the same there is some effort made to educate and recognize personal ability. There are classes of instruction given to the Infirmarists by the doctors-on nursing subjects mostly, as far as we could learn, and with just enough anatomy thrown in to make such instructions have a practical bearing. We saw the room where these lectures (they are not classes in our sense of the word) were given; it was a tidy little room, containing about a dozen chairs, and all four walls covered with shelves full of pathological specimens in jars. By means of these lectures the Infirmarists were taught how to do various forms of treatment, which otherwise would be left for the doctor himself to do, so that perhaps the motives that inspire such instruction are not wholly unselfish, but, nevertheless, it is a recognition on the physician's part of a requirement for nursing skill and efficiency that has hitherto never been felt. It is a beginning step in the right direction, and a voluntary one at that.

After her admission to the hospital the Infirmarist must spend six months in the kitchen learning to cook; then she goes to the wards and to class, and she must go to class always after that until she learns enough to be allowed to stop. Some of them have been going for a long time, while some newcomers, possessed of more capacity, are excused after a few months' attendance: some of the Infirmarists, we heard, after being in the hospital only a very short time, a few weeks in some cases, are by reason of their ability promoted to the maximum salary, while there are several instances of those who have been in the hospital for fifteen years, or ever since its foundation, and who are only receiving the lowest, that is, twenty-five lira (five dollars) a month. These unfortunates must always go to class!

In the wards too the women nurses wear a sort of uniform of gingham; they also wear white muslin caps, from the back of which float two white strings about two inches wide and about a yard long. The condition in which they keep the wards was above reproach-the brass and glass and instruments fairly shone with polishing, and the patients themselves all seemed comfortable and at least looked clean. It seemed, indeed, as if in this, said to be the most modern and progressive hospital in Italy, where medical service and surgical technique are outclassed by none, that the day will soon come when the nursing standards will be on a par with the beautiful mechanical equipment which make it one of the finest institutions on the Continent. 